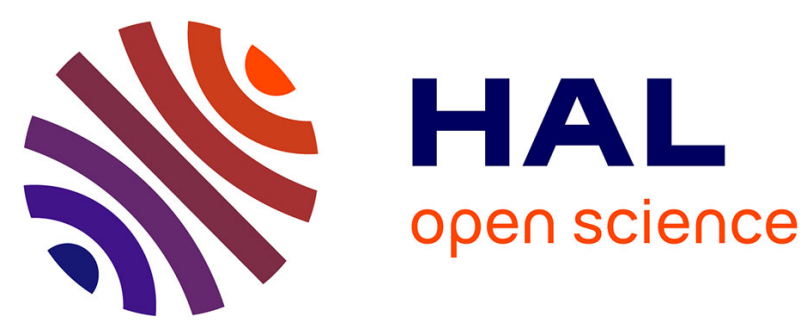

\title{
Anorexia nervosa during adolescence and young adulthood: Towards a developmental and integrative approach sensitive to time course.
}

Ludovic Gicquel

\section{- To cite this version:}

Ludovic Gicquel. Anorexia nervosa during adolescence and young adulthood: Towards a developmental and integrative approach sensitive to time course.. Journal of Physiology - Paris, 2013, 107 (4), pp.268-277. 10.1016/j.jphysparis.2013.03.010 . inserm-00818227

\section{HAL Id: inserm-00818227 https://www.hal.inserm.fr/inserm-00818227}

Submitted on 26 Apr 2013

HAL is a multi-disciplinary open access archive for the deposit and dissemination of scientific research documents, whether they are published or not. The documents may come from teaching and research institutions in France or abroad, or from public or private research centers.
L'archive ouverte pluridisciplinaire HAL, est destinée au dépôt et à la diffusion de documents scientifiques de niveau recherche, publiés ou non, émanant des établissements d'enseignement et de recherche français ou étrangers, des laboratoires publics ou privés. 
Anorexia nervosa during adolescence and young adulthood:

Towards a developmental and integrative approach sensitive to time course

L. Gicquel

INSERM U 894 Team 1, Centre de Psychiatrie et de Neurosciences, Paris, France

Pôle Hospitalo-Universitaire de Psychiatrie de l'Enfant et de l'Adolescent, Université de Poitiers, Centre Hospitalier Henri Laborit, France

Address: Pôle Hospitalo-Universitaire de Psychiatrie de l'Enfant et de l'Adolescent, Centre Hospitalier Henri Laborit, CMPEA, 7, allée des anciennes serres, Saint-Benoît 86280, France. Tel.: +33 5495234 92; fax: +33 549523494

E-mail address: ludovic.gicquel@ch-poitiers.fr 


\section{Abstract}

Anorexia nervosa is situated at the junction between two developmental periods: adolescence, where intense physiological and psychological upheavals are occurring over a relatively short period of time, and the potentially chronic nature of the disease over the course of the patient's lifespan. This second, broad time window links on one hand the critical period of adolescence with the pre-morbid period, during which a complex state of vulnerability, often unseen and unheard, combines with different risk factors (which may be isolated, associated, dissociated or concomitant) to produce the emergence of anorexia; on the other hand it ushers adolescence into the period of adulthood, flagged with the reorganization that occurs in the course of the healing process (in case of recovery), or pervaded by somatic and mental distress (in cases where the condition becomes chronic). Given the lifespan nature of the disease, it is difficult to differentiate premorbid pathogenic factors from changes resulting from the acute or chronic phases of the illness. It is also difficult to establish straightforward correlations between physiological disturbances and their clinical consequences, or conversely to assume that the restoration of physiological parameters means the disappearance of the underlying mental disorder. Taken together, these observations support an approach to anorexia nervosa that is both developmental and integrative, taking into account both the complexity of the pathways involved and the developmental timescales of these pathways. This type of approach can help to adjust therapeutic strategies and thus enhance prognosis, in particular by integrating the temporal parameter into the dynamics of care plans. 


\section{Introduction}

Ackard et al. (2007) found that $34 \%$ of American boys and $43.5 \%$ of American girls show eating disorder (ED) traits during childhood or adolescence. During the adolescent critical period, some of these children will develop sub-threshold or/and threshold anorexia nervosa (AN). Lifetime prevalence of AN is approximately $0.6 \%$ overall (Jacobi et al., 2004; Hudson et al., 2007), 0.3\% in men and 0.9\% in women (Kessler et al., 2004, 2005), and AN may affect up to $1 \%$ of adolescents and young adults (90\% of these being girls) aged between 15 and 24 years. The highest incidence rate subgroup for AN includes adolescent girls aged 15 to 19 and accounts for about $40 \%$ of all identified cases (Hoek and Hoeken, 2003). Lucas et al. (1999) reported a continual rise in incidence rates since the 1930s, with a maximum for the period 1980-1989. Throughout the period studied (1935-1989), the increase was most marked among females aged 15-24. The incidence of AN in Europe seems to have remained stable since the 1970s (Hoek, 1993). In many ways, adolescence appears to be the crucial period for the development of this complex developmental disorder. Apart from these epidemiological data, most clinical studies on AN have been cross-sectional or retrospective, rarely longitudinal, and have focused on specific parameters, such as population types, symptom characteristics or outcomes. AN can also be considered in terms of a timeline progression from vulnerability, onset, sub-/threshold syndromes, diagnosis, acute phase, recovery, relapse, remission, and finally chronic evolution or death. These elements subsume the main phases in the course of AN and highlight a developmental and evolving process that most frequently occurs during a vulnerable period of fundamental change.

What does vulnerability mean in this context? Which modifications or alterations precede the emergence of the illness and which are the consequences of it? Which factors 
account for its intractability or, conversely, for recovery? Does the duration of the illness account for its outcome?

More and more, it appears that the way in which symptoms follow each other temporally is important, from onset to chronic AN. Following the multifactorial model of AN (Garner, 1993), which proposes the existence of predisposing, precipitating and perpetuating factors, numerous studies now approach AN pathogenesis from a developmental and integrative point of view.

This article presents a review of the literature focused on AN development and its evolution from a perinatal vulnerability period to chronic illness during adulthood, from onset to recovery, from acute phase to weight restoration. It also explores psychological and physiological factors, as well as risk factors and pathogenesis models.

\section{Developmental Risk factors for anorexia nervosa}

In a recent seminar, Treasure et al. (2010) envisaged the pathogenesis of eating disorders (ED) as being comprised of three main types of risk factors: genetic factors, biological factors and environmental context. The Academy for Eating Disorders considers ED to be biologically based forms of severe mental illnesses. They also briefly explored interactions between the environment and individual biology. An exhaustive review of risk factors for ED conducted by Strigel-Moore and Bulik (2007) and a recent article by HerpertzDahlmann et al. (2011) about the etiology of AN have painted a broad picture of the main risk factors.

To broach a recurrent theme in the present article, we will first consider from among this broad set of risk factors the perinatal factors. A wide range of factors can increase the risk of $\mathrm{AN}$, from genetically mediated maternal variables that can influence the intrauterine environment (Strigel-Moore and Bulik, 2007) to neonatal complications. On the maternal 
side, Favaro et al. (2006) reported that maternal anemia, diabetes mellitus, preeclampsia and placental infarction were all significant independent predictors of later occurrences of AN. Shoebridge and Gowers (2000) explored exposure to stress during pregnancy. Bloomfield et al. (2003) noted that moderate maternal food restriction around the time of conception results in an early fetal cortisol surge and risk for preterm birth. Strigel-Moore and Bulik (2007) hypothesized that preterm births and small-for-gestational-age (SGA) babies were more likely to experience the persistence of subthreshold AN symptoms and continuing efforts toward food restriction and weight control which "flew under the radar". Along the same lines, Norre et al. (2001) emphasizes the possible underestimation of eating disorders in fertility clinics. In women with prolonged food-deprivation, delivery is more likely to occur at an earlier gestational age, and it is potentially mediated by higher corticotrophin-releasing hormone levels (Hermann et al., 2001). For other researchers, birth-related complications such as prematurity (SGA) and cephalhematoma were found to be associated with increased risk for developing AN later in life (Cnattingius et al., 1999). Likewise, cardiac problems and hyporeactivity were also found to be significant independent predictors of the subsequent occurrence of AN (Favaro et al., 2006) and subtle brain damage at birth (as a consequence of hypoxia) could result in early feeding difficulties. Thus, Strigel-Moore and Bulik (2007) concluded, "An intricate cycle of genetic factors (influencing weight gain during pregnancy) and environmental factors (foetal undernutrition in utero) coupled with the direct heritability of ED could jointly act to influence eating disorder risk in offspring".

As a prelude to their presentation of genetic factors, Treasure et al. (2010) outlined that $\mathrm{AN}$ is a complex genetic disease and that it is at the crossroads between approaches focusing on comorbidities and a developmental approach that takes into account different combinations of genetic risks, such as ED and depression (Wade et al., 2000), ED and anxiety disorders (Keel and Klump, 2005), and ED and addictive disorders (Baker et al., 2007). An overview of genetic influences in AN was proposed by Striegel-Moore and Bulik (2007) and includes three possible models. First, there is a gene - environment interaction model. This model postulates that different genotypes could influence individuals' sensitivity 
to environmental events. Second, there is a model, which includes major genetic effects, affecting processes of appetite and weight dysregulation. Finally, the authors presented a mixed model, which takes into account the fact that "our diagnostic categories may be heterogeneous mixtures of genetically influenced symptoms and cultural overlays that may not accurately reflect underlying biology". In other words, Striegel-Moore and Bulik (2007) favor a model whereby core symptoms of ED are mainly governed by genetic effects. Along the same lines, Grice et al. (2002) considers the core phenotype of persistent low body weight to be genetically underpinned, alongside a notion that "the pervasive culture of the times may have filled in the gap by providing an explanatory context and padding the definition of syndromes with environmentally mediated and contextually plausible symptoms".

Such genetic explorations have been mainly based upon twin and molecular genetic studies although, as suggested by Striegel-Moore and Bulik (2007), it would be of interest to explore genetics of the course of the illness. The lifetime risk for first-degree relatives of patients with AN for developing an eating disorder themselves is 7 times higher than in the families of healthy controls (Herpertz-Dahlmann et al., 2011). According to studies of twins, the heritability of AN ranges from $48 \%$ (Kortegaard et al., 2001) to $76 \%$ (Klump et al., 2001). In addition, the weight of environmental factors alone was greater than the weight of a shared environment (Striegel-Moore and Bulik, 2007).

Molecular genetic studies have been comprised mainly of genetic association and linkage studies. Typically, candidate gene studies have been performed on particular neurotransmitters of interest, such as dopamine, serotonin and opioids. Neuropeptides, which are substances related to brain development and the neuroendocrine system, have also been also explored. Although Bergen et al. (2003) showed a significant association between AN and serotonin 1D (HTR1D) and delta opioid (OPRD1) receptor genes, another recent study found no statistically significant associations for any individual SNP or haplotype block with any definition of the illness. 182 candidate genes and 5,151 SNPs were studied. (Pinheiro et al., 2010). The population of this collaborative study consisted of 1085 AN 
patients and 677 controls. Three subtypes of AN (any subtype, no binge eating behavior, restricting AN) were defined to perform separate association analyses.

Helder and Collier (2011) used genetic linkage studies to implicate chromosome 1 (1p33-36; 1q41) in AN. In 2002, in a study of restrictive AN patients, Grice et al. had already proposed a susceptibility locus on this chromosome. Accordingly, Devlin et al. (2002) focused on "key behavioral covariates (drive for thinness and obsessionality)" and proposed certain regions of interest on chromosomes 1, 2 and 13. Nevertheless, in a recent large AN population study, Wang et al. (2010) did not identify any genome-wide signals.

The main difficulties in identifying biological risk factors for AN consist of differentiating anomalies causally associated with AN before its onset from the consequences of the starvation process itself in the acute phase or from biological disturbances over the chronic course of AN. Treasure et al. (2010) noted that poor nutrition induces both a general effect on brain function and a specific effect on the appetite system. Dysregulation of central control over the appetite is of great interest in AN pathogenesis. Marsh et al. (2009) suggested that abnormal changes in three systems (Homeostatic system, Drive system and Self-regulation system) play a role in the initial occurrence and long-term persistence of ED. AN onset could be linked to "pervasive deficits in self-regulatory systems". Treasure et al. (2010) suggests that social influences (stigmatization of fatness, idealization of thinness) could lead to weight control behaviors and produce a destabilizing effect on the biology of appetite control. Miller (2011) proposed four etiological pathways for endocrine dysfunction in AN: heritability, appetite dysregulation, reward circuit dysregulation and neuromediator dysregulation. There is increasing evidence for appetite hormone dysregulation, and Miller (2011) speculated that elevation of the anorexigenic hormone PYY could be implicated in AN. She also suggested a possible resistance to the effects of ghrelin in AN, but the few available studies supporting this hypothesis are controversial.

Recently, Kaye and Bailer (2011) published a commentary in Biological Psychiatry entitled "understanding the neural circuitry of appetite regulation in ED" regarding the study by Gérard et al. (2011). Gérard et al. (2011) explored the role of the endogenous 
endocannabinoid system in AN. This commentary echoed another article, published by Kaye et al. in 2009 entitled "New insights into symptoms and neurocircuitry function of AN", wherein the neurocircuitry of appetite was detailed. In both studies, the insular cortex, and more particularly the anterior insular cortex, was investigated because of its many functions in hunger and satiety, and because of its involvement in interoceptive processing. Kaye et al. (2011) suggested that the "anterior insula response to palatable food codes a biosignal related to the sensory-interoceptive aspects of appetite behaviors and translates this signal into the motivation to eat". Gérard et al. (2011) found an increase in CB1 receptors in the insular cortex in AN. Although this increase may be one of the many malnutrition-induced compensatory responses present in AN, it also supports a pathogenesis hypothesis whereby endocannabinoid pathways could contribute to abnormal hedonic input into sensory/interoceptive/motivation signals.

Some authors have conducted studies between the perinatal period and adolescence (i.e., during childhood) and have focused on personality traits the predict AN emergence. Herpertz-Dahlmann et al. (2011) suggest that anxiety (frequently comorbid with AN) "commonly predates the eating disorder and has its onset during childhood". Separation anxiety and social anxiety are the two main types of AD reported in childhood in AN patients. Other traits, such as dysphoric mood and poor interoceptive awareness, could also be predisposing factors for later AN (Herpertz-Dahlmann et al., 2011). Finally, Rastam (1992) proposed rigidity, obsessiveness and perfectionism (symptoms of the autism spectrum disorders) as childhood risk factors for AN because these traits are robust in adolescents and adults with AN.

In the development of individuals who will later suffer from AN, puberty and the postpuberty years are crucial periods of vulnerability (Treasure et al., 2010). During this period, genes involved in genetic risk could be "switched on" (Herpertz-Dahlmann et al., 2011). Modifications in the hippocampus and amygdala, which are associated with the development of mood and anxiety disorders, often present during the acute phase of $\mathrm{AN}$, but they are also common throughout the course of eating disorders. Likewise, Neufgang et al. (2009) 
emphasized links between "sexually dimorphic brain areas", Tanner stage and the level of circulating steroid hormones. The same year, Kaye et al. (2009) wrote, "gonadal steroids directly alter affective processing as well as neurotransmitters (known to play a significant role in the pathophysiology of eating disorders) such as dopamine, serotonin, opioids, oxytocin and vasopressin. In other words, puberty implies for everyone profound and varied modifications in different areas of human functioning". The pubertal maturation phase, combined with different vulnerability factors, might see the start of a silent process that could lead to sub-threshold and/or threshold symptoms of AN.

Western idealization of thinness highlights the role of cultural influences as risk factors for ED, and especially for AN, during adolescence. Striegel-Moore and Bulik (2007) devoted a long passage to cultural risk factors in ED. They explored socioeconomic status, and the influence of race/ethnicity, and they focused on the concept of internalization of the thin beauty ideal (Rodin et al., 1985). The authors also emphasized the importance of cultural factors in the increased incidence of ED. Environmental changes are more appropriate to explain this increase because the "gene pool cannot change that quickly" even if "consideration of other mechanisms of gene-environment interplay" should moderate these remarks. The overrepresentation of Caucasians among women with AN was noted by Kessler et al. (2005), and they suggested that sample bias might interfere with a size effect. By contrast, a consensus has been reached that female gender is a fixed and potent marker for AN (Jacobi et al., 2004, Treasure et al., 2010).

Body image is subjected to different realities and influences. In one American study, $47 \%$ of girls enrolled between the fifth and the twelfth grades reported a desire to lose weight as a result of magazine photos, while another $69 \%$ of girls agreed that magazines have an influence on their image of the ideal body shape (Levine, 1998). Body image, body weight, body dissatisfaction, body symptoms, the thin ideal, and other such concerns place the body at the core of AN. Along these lines, Rodin et al. (1985) suggest that internalization of the "thin beauty ideal" increases the risk for ED symptoms. This ideal, alongside body dissatisfaction, excessive body weight and dieting, are risks for the onset or deterioration of 
an eating disorder (Mc Knight Investigators, 2003). The influence of the media and social pressures, not far removed from the "thin ideal," are naturally questioned. To what degree does this reflect reality? Is the influence overestimated? Striegel-Moore and Bulik (2007) suggest that these factors "increase body-image concerns at least in the short run".

Reflections by Striegel-Moore and Bulik (2007) conclude our discussion of risk factors. They propose that "component symptoms are more common than full syndromes" and that "it cannot be assumed that the risk profile for a symptom necessarily reflects the risk profile for the entire syndrome". The composite profile of AN includes biologically mediated symptoms (such as low body weight) vs. environmentally mediated symptoms (such as selfevaluation and body image) (Striegel-Moore and Bulik, 2007), and the heterogeneity of risk factors highlights the "many risk pathways toward ED". In addition, as risk factors can be single or multiple and can appear successively or in combination, their clinical consequences might be different.

Finally, when all components of vulnerability are present when the developmental period is favorable (i.e., post-puberty physiological and psychological modifications co-occur with increasing societal demands; Herpertz-Dahlmann et al., 2011) and when precipitating factors occur (Garner, 1993), AN sub-threshold or threshold symptoms occur. Some warning symptoms may also be present at an earlier stage in young adolescent girls, such as disturbed menstruation or excessive preoccupation with the body. 


\section{Acute phase of the illness}

Among the acute symptoms of AN, the DSM-IV-TR (1994) focuses on four specific criteria to make a diagnosis of current AN. Weight is a central component in the diagnosis, with refusal to maintain body weight, intense fear of gaining weight and disturbances in the way in which the subject's body weight is experienced as primary symptoms. Body shape is also important. Amenorrhea is the only "physiological" criterion for a diagnosis of AN. The distribution between psychological and physiological symptoms most likely undervalues the role of physiological factors, all the more so because some psychological symptoms seem to be underpinned by physiological parameters. Thus, without extensively rehashing the main clinical symptoms of $\mathrm{AN}$, we will focus on associated physiological factors that determine onset of the acute phase and could have an effect on the time course (e.g., the maintenance or recovery) of $\mathrm{AN}$.

Nevertheless, as a prelude to biological considerations, we will present certain neuropsychological factors associated with AN. In adolescent patients, deficits in set-shifting are not only pronounced but also correlate with perfectionist traits (Bürhen et al., 2012). Gillberg et al. (2010) proposed a new approach to certain AN symptoms by suggesting an overlap between AN and Autistic Spectrum Disorders (ASDs), which have corresponding cognitive styles including impaired set-shifting, weak central coherence and an impairment in theory of mind (Herpertz-Dahlmann et al., 2011). Importantly, these impairments are independent from the state of starvation, and they are stable characteristics of individuals with AN.

Biological and endocrine dysregulation and brain dysfunction will be presented in light of the AN starvation process. The process is dynamic and most likely involves differences between the acute and non-acute phases, such that any biological parameters may vary from onset to chronic evolution of the illness. In addition, some dysfunctions are most likely causally associated with AN where others are only its consequences, and neurochemical abnormalities do not necessarily involve functional or clinical consequences. Therefore, 
because data are varied and numerous, the following results should be considered cautiously.

With regard to biological anomalies, Hasan and Hasan (2011) explored the dysfunction of various neuropeptides associated with AN (at both the central and peripheral level), and they focused especially on corticotrophin-releasing factor (CRF), opioid peptides, ghrelin and leptin. The authors performed an exhaustive literature review and developed the concept of a "unified neurological perspective" for AN. The main characteristics of this model will be presented later in the paper. Nakazato et al. (2009) showed that concentrations of brainderived neurotrophic factor in the blood are reduced in acute AN, and Ribases et al. (2005) proposed a trait-related disturbance in the system on the basis of genetic studies.

Endocrine dysregulation in AN involves the gonadal axis, GH resistance, the skeletal system, and the hypothalamic-pituitary-adrenal (HPA) axis, with axis interrelations. Dysregulations in the gonadal axis include a decrease in LH pulsatility and in estrogen and androgen levels (Miller, 2011). Miller (2011) focused on amenorrhea and androgen deficiency. She reiterated that the "degree of luteinizing hormone (LH) suppression did not correlate reliably with duration of illness or degree of thinness, and return of menses did not demonstrate a simple relationship to weight". She noted the absence of a predictive leptin cutoff level and highlighted the important role of this hormone in the development of hypothalamic amenorrhea in women. She cautiously pointed to a future need for studies "to determine the pathophysiology of amenorrhea in AN". Similarly, Miller (2011) underlined that the effects of androgen deficiency (a consequence of hypogonadotrophic hypogonadism) in women with AN are largely unknown. Miller et al. (2007) suggest that this deficit could increase the severity of mood disorders in patients with AN. Miller (2011) also supports the established role of $\mathrm{GH}$ as a mediator for lipolysis, independently from IGF-1. IGF-1 levels are strong predictors of abnormal bone microarchitectural parameters (Lawson et al., 2010). 
There is a functional link between the gonadal axis and the skeletal system because "the normalization of reproductive function is the strongest predictor of skeletal recovery, and recovery of menses predicts an increase in bone mineral density independent from changes in weight" (Miller et al., 2006). These remarks raise the issue of skeletal abnormalities associated with AN. Grinspoon et al. (2000) found that bone loss is an important outcome of AN in women (90\% of young women with AN present a significant decrease in T score), and AN is characterized by a concomitant decrease in bone formation and increased resorbtion (Grinspoon et al., 1996). The consequences of AN on bone structure are all the more important because adolescence is a critical period both for the onset of $\mathrm{AN}$ and for rapid bone mass accrual. Similarly, Soyka et al. (2002) demonstrated that adolescent girls with AN do not experience the usual linear increase in bone mass during puberty. Misra et al. (2004) concluded that adolescents with AN presented lower bone mineral density in the spine and hip compared to healthy adolescents of comparable skeletal age. As peak bone mass is likely to be lower than expected, later consequences are the development of osteoporosis and fractures. Rigotti et al. (1991) found fracture risk to be seven times higher in AN than expected for age and gender, and Miller et al. (2005) concluded that the combination of low bone mineral density (potentially worsened by excessive load from exercising - Waugh et al., 2011) abnormal skeletal microarchitecture and reduced bone strength was associated with a fracture rate of $30 \%$. Prior to the decrease in bone mineral density, Bredella et al., (2008) noticed that very early in the course of AN in adolescent girls, bone volume and trabecular thickness were already abnormal. In contrast to generally accepted ideas, Hadigan et al. (2000) found that "vitamin D and calcium intake are comparable in women with AN and healthy women of comparable age and therefore they are not likely to be important pathogenic factors". Finally, Miller (2011) reached no conclusion between a causal implication of bone marrow fat increase in bone loss and osteoblasogenesis with default into the fat lineage.

Along this line, the main characteristic of hypothalamic-pituitary-adrenal axis dysregulation in adolescents with $\mathrm{AN}$ is hypercortisolemia (Misra et al., 2004), and higher 
overnight cortisol levels predict weight gain (Misra et al., 2006). In addition, Lawson et al. (2009) found that overnight blood cortisol levels in women with AN were inversely associated with bone mineral density and positively associated with the severity of depression and anxiety symptoms. Therefore, Miller (2011) concluded that "hypercortisolemia may also contribute to the severe bone loss incurred, and the highly prevalent psychiatric comorbidity in women with AN".

The brain is implicated in the psychological and physiological processes occurring in onset maintenance and recovery of AN. Interactions between symptoms, the starvation process and the brain throughout the duration of AN lead to important modifications or damage in central and peripheral metabolism that can affect $\mathrm{AN}$ outcome. It is difficult to differentiate developmental brain modifications (especially during the vulnerable period of brain restructuring during adolescence) from AN brain alterations and to distinguish structural alterations brought about by the illness from functional consequences that potentially accompany symptoms.

In 2011, Jáuregui-Loreba reviewed studies of neuroimaging in ED and, more particularly, those focused on early-onset AN, brain change and dysfunctions neuroimaging or neurotransmitters. Another review, published by Van den Eynde et al. (2012), included studies of structural magnetic resonance imaging (MRI) in ED and, more particularly, imaging during the acute phase in adolescents with AN. Structural neuroimaging includes both computed tomography (CT) and magnetic resonance imaging (MRI) and affords the study of brain anatomy. Functional neuroimaging includes positron emission tomography (PET), single photon emission computed tomography (SPECT) with regional cerebral blood flow focus, magnetic resonance spectroscopy and voxel-based morphometry (VBM).

Herpertz-Dahlmann et al. (2011) found that adolescents with AN-related starvation had an overall loss of brain volume. Gaudio et al. (2011) found a comparable result in the early stages of the illness using optimized voxel-based morphometry in a population of adolescents with AN-restricting type. Herpertz-Dahlmann et al. (2011) found support for the starvation hypothesis in the fact that brain volume deficits were associated with metabolic 
energy reduction due to poor nutrition. Chui et al. (2008) found that the correlation between elevated serum cortisol and the loss of grey matter (GM) was independent from changes in BMI.

Beyond a global brain volume decrease, Herpertz-Dahlmann et al. (2011) estimated a 5 to $20 \%$ loss of GM in AN. White Matter (WM) volume has not been widely investigated, and one study found no indication of abnormalities (Jáuregui-Loreba, 2011). GM loss appeared to be more marked in some areas. Bilateral region-specific decreases in GM volume were found by Gaudio et al. (2011) at onset of AN in the middle cingulate cortex, the precuneus, and the inferior and superior parietal lobules. McCormick et al., (2008) also noticed a specific decrease in volume of the anterior cingulate cortex, the hippocampus and the temporal, parietal and prefrontal regions. Although Castro-Fornieles et al. (2009), like Gaudio et al. (2011), found that a wide range of brain areas had low GM volumes in adolescents with AN, there was no clear overlap between these regions in the two studies.

A recurrent finding from SPECT studies is of unilateral blood flow hypoperfusion in AN $(73 \%$ of children and adolescents with early-onset AN had asymmetry in the blood flow in at least one area of the brain; Chowdhury et al., 2003), and more specifically hypoperfusion of the temporal lobes specifically (Gordon et al., 1997; McCormick et al., 2008). JimenezBonilla et al. (2009) found that temporal hypoperfusion in the acute phase was correlated with the long-term clinical outcome in patients with ED, suggesting a prognostic value of temporal hypoperfusion. Almost $75 \%$ of patients with early-onset of AN showed no association between temporal unilateral blood flow reduction and cerebral dominance, nutritional status, length of illness, mood, or eating psychopathology. Likewise, JáureguiLoreba (2011) reported that seven years after onset of AN, reduced blood flow in the temporal region still affected two thirds of patients, and no significant correlation between this reduction and body mass index, minimum BMI, residual eating disorder psychopathology, or intelligence quotient was found. These results naturally cast doubt on relationships between morphological variations in the setting of AN and their functional predictive value. Conversely, Lask et al. (2005) noticed a significant association between temporal blood flow 
reduction and impaired visuospatial ability, impaired complex visual memory, and enhanced information processing. Likewise, McCormick et al. (2006) reported a similar association between reduction of right dorsal anterior cingulate cortex volume and lower performance on IQ tests. Finally, Suda et al. (2010) stated that a tendency towards dieting correlated with blood flow in the right frontotemporal cortex and that dietary behavior problems correlated with the left orbitofrontal cortex.

Functional MRI studies have focused mainly on body image or perceptions, although some have explored stimulation derived from food. Abnormal activity in the insular and orbitofrontal cortex, as well as in other regions including the mesial temporal and parietal regions and the anterior cingulate cortex, was found when pictures of food were presented to AN patients (Nozoe et al., 1993, 1995; Ellison et al., 1998; Naruo et al., 2000; Gordon, 2001; Uher et al., 2004).

Beyond standard DSM criteria, moderate to severe body image disturbances appear to be core symptoms in AN, and, unsurprisingly, a large number of studies have explored this theme. Parietal cortex mediates perceptions of the body, and disturbances in the left hemisphere may contribute to body image distortion (Jáuregui-Loreba, 2011). Boghi et al. (2011) suggested that brain atrophy in temporoparietal areas could account for body image distortion. Using PET, Bailer et al. (2004) found a negative relationship between 5HT2A receptor activity in the left parietal cortex, the right occipital cortex, and the left subgenual cingulate cortex and scores on the subscale of "drive for thinness" (EDI). Mohr et al. (2010) proposed an additional role for the insular and lateral prefrontal cortex. AN patients reported stronger satisfaction ratings in response to images corresponds with the thin idea, and showed stronger activation of these brain structures in response to these ratings. This suggests AN patients experience stronger emotional involvement when the subject is presented with distorted images close to their own ideal body size. Finally, Beato-Fernandez et al. (2009) concluded that functional brain abnormalities in patients with AN might be related to the storage of a distorted prototypical image of the body in the left parietal lobe, 
and activation of the right temporal area after exposure to images of patients' own bodies might be consistent with this adverse response.

There is also an evident attentional bias towards food and body shape in AN patients, and this bias could be associated with increased activation in disturbed neural networks involved in self-regulation and hedonic motivation (Kaye et al., 2009; Van den Eynde and Treasure, 2009). Therefore, in addition to body-image disturbances, functional neuroimaging studies have also considered neuropsychological impairments or deficits in AN patients. Herpertz-Dahlmann et al. (2011) reported that their sample of patients with eating disorders had a strong attentional bias towards eating and weight stimuli, which did not always normalize after weight recovery. Impaired cognitive flexibility, impaired cognitive set-shifting abilities and deficits in social cognition were shown by other authors to be independent from nutritional and body weight status in AN adults. These disturbances are partially associated with hypoactivation in the ventral anterior cingulate-striato-thalamic loop, which is known to be involved in motivation-related behaviors (Friederich and Herzog, 2011).

\section{Weight restoration phase and recovery}

Castro-Fornieles et al. (2009) reports that many symptoms resolve with weight gain and when brain mass is restored. Is weight restoration synonymous with brain restoration? Is brain restoration synonymous with recovery? Is the AN subtype of importance? How can brain modifications - both primarily and secondarily associated with AN - be distinguished from developmental changes during adolescence? What is the effect of illness severity? As some aspects of AN pathogenesis are weight independent, what does weight normalization mean? In the words of Jáuregui-Loreba (2011), "the interaction among low weight, duration of illness, and brain changes remains controversial to some extent".

Neuroimaging studies contribute to a better understanding of weight restoration and recovery phenomena. Using VBM in AN adolescents, Castro-Fornieles et al. (2010) noted that GM alterations were reversible after nutritional recovery and that GM and WM both 
increased significantly in volume during weight restoration. Overall, Herpertz-Dahlmann et al. (2011) found that most of the brain reduction is reversible following weight restoration, and they found a regeneration of $60 \%$ of the deficit within 15 weeks in their study. McCormick et al. (2006) linked a "smaller change in right dorsal anterior cingulate cortex volume" to relapse outcomes instead of recovery outcomes. In addition to weight loss intensity, the duration of AN is also of importance for brain functioning. Boghi et al. (2011) recently found that the cerebellum was proportionally more affected when disease duration was longer, whereas a short period of AN was associated with hypothalamic alteration. The authors conclude that cerebellar GM alterations "could play a role in the chronic phase of the illness".

Frampton et al. (2011) found that blood flow in the medial temporal region did not return to normal after weight restoration. Likewise, Gordon et al. (1997) noticed the persistence of temporal hypoperfusion and cognitive distortions, and suggested that hypoperfusion might not be directly related to weight loss. Komatsu et al. (2010) found a significant positive correlation between $\mathrm{BMI}$ and regional blood flow in the right thalamus, the right parietal lobe, and the right cerebellum. Frank et al. (2007) additionally found that regional cerebral blood flow normalizes with long-term recovery.

As suggested by Wagner et al. (2006), the choice of criteria used for defining recovery is relevant when the aim is a comparison or meta-analytic review. Bailer et al. (2007) defined recovery as the combination of normal body weight maintained for more than one year, regular menstrual cycles and the absence of binging or purging behaviors. The degree and duration of starvation have an effect on recovery outcomes and AN persists more when these symptoms also persist (Treasure et al., 2010). Papadopoulos et al. (2009) found that somatic and psychiatric comorbidity was associated with poor outcomes. By contrast, young age at onset and short duration of AN were associated with a good outcome.

Even if recovery is generally accompanied by the global restoration of GM volume (without any significant association between BMI and brain tissue volumes), Van den Eynde et al., (2012) points to the lingering persistence of specific GM abnormalities. Nevertheless, 
as their evaluations occurred after only 6 months of treatment, the authors did not exclude the possibility of full recovery with a longer follow-up. Concerning regional GM abnormalities, as is the case for starvation, weight gain might activate specific brain regions that are possibly relevant to the pathophysiological aspects of the disorder (Jáuregui-Loreba, 2011). In patients who had recovered from restrictive $\mathrm{AN}$, one study reported that the dorsal raphe nucleus and the anteroventral striatum presented a greater binding potential compared with purging $\mathrm{AN}$ and that the mesial temporal and parietal cortical areas and the subgenual and pregenual cingulate cortex were, for their part, associated with a lower binding potential (Frank et al., 2002). Likewise, GM volume in the ACC was found to be abnormal both in the acute phase and during recovery (Joos et al., 2010, Mülhau et al., 2007). Uher et al. (2003) concluded that separate neural correlates underpin trait and state characteristics of AN. The medial prefrontal response to disease-specific stimuli may be related to trait vulnerability. Lateral and apical prefrontal involvement is associated with a better outcomes. McCormick et al. (2008) found that volume loss in the ACC was inversely correlated with the level of perceptual organization and reasoning skills, and predicted worse outcomes of the eating disorder 1 year later. Jimenez-Bonilla et al. (2009) additionally found that temporal hypoperfusion in the acute phase is correlated with the long-term clinical outcome in patients with ED, suggesting a prognostic value of temporal hypoperfusion. In an 18-year follow-up study of adolescent AN subjects, difficulties in mental processing tasks remained in several subjects after recovery, and these deficits were independent of body weight loss and the duration of the eating disorder (Wentz et al., 2009).

In addition to structural brain modifications, brain monoamine function was studied in AN during the acute phase and after recovery by Kaye et al. (2009). The authors stated that " $5 \mathrm{HT} 2 \mathrm{~A}$ receptors are reduced and $5 \mathrm{HT} 1 \mathrm{~A}$ receptors are increased in both the acute and recovery state, and dopamine receptors (DA2) within the striatum are increased after recovery". Fox (2008) explored these alterations and noted that they were persistent during recovery in the frontal, subgenual cingulate, and mesial temporal regions. He suggested a role for the ventral limbic system. It is interesting to note that a recent association between 
the normalization of sex hormones and an increase in GM in limbic brain areas was found in weight-recovered adolescent AN patients (Herpertz-Dahlmann et al., 2011).

Jacoangeli et al. (2006) noticed the persistence of amenorrhea in approximately $15 \%$ of women despite weight recovery. A combination of residual psychological symptoms, lower leptin levels and lower fat mass may explain this phenomenon (Brambilla et al., 2003). For skeletal recovery (reversal of bone loss), the spontaneous return of menstruation is more important than weight gain per se (Miller et al., 2006; Dominguez et al., 2007). Bone mineral density improves with recovery from AN, but does not return to normal (Herzog et al., 1993). Gwirtsman et al. (1989) associates normalization of hypercortisolemia with long-term weight normalization.

\section{Chronic evolution of anorexia nervosa}

The acute phase can be followed by remission and relapse, by remission, relapse and recovery, by remission, relapse, and chronic illness or by chronic illness without any remission, from adolescence to adulthood. In some cases, a vicious cycle crystallizes between premorbid traits and the consequences of the illness which will then worsen the course of AN.

Even if most adolescents and young adults with AN will recover (fully or partially) from $57.1 \%$ to $85 \%$ (Steinhausen, 2002; Johnson et al., 2002) - chronic illness persists in $17 \%$ of patients (Steinhausen, 2002). The rate of mortality is almost $5 \%$ among young patients with AN (suicide ...) and rises to $20 \%$ after 20 years. After the acute phase and before full or partial recovery, the relapse period has been explored. According to Carter et al. (2004), the overall relapse rate in $\mathrm{AN}$ is $35 \%$. Most relapses occur between six and seventeen months after discharge, and the authors pinpoint five key predictors for relapse: history of previous treatment, history of suicide attempts, associated OCD symptoms at presentation, concern for body at discharge and initiation of excessive physical activity after discharge. 
Some studies highlight factors that could contribute to chronic evolution of AN. In line with Garner's (1993) concept of perpetuating factors, Herpertz-Dahlmann et al. (2011) proposed a specific combination of factors (long-standing malnutrition during adolescence and young adulthood, hormonal and neuropeptide dysfunctions) that "may produce biological scars that maintain and accelerate the disorder and likely result in chronic mental disorders". Schmidt and Treasure (2006) grouped the effects of nutritional deficits into four broad domains (after Treasure et al., 2010): medical effects on the body and brain, interpersonal effects, exacerbation of avoidant coping, and obsessive compulsive traits. Gillberg et al. (2010) proposed that autistic traits in childhood may be predictive of poorer global outcomes of the eating disorder in adulthood. Chui et al. (2008) found an association between amenorrhea in AN with chronic evolution and certain cognitive deficits (recall, verbal memory, working memory, visual reproduction, reading, math and oral language).

Liao et al. (2009) found difficulties in functional decision-making could also contribute to the chronic course of AN. Wagner et al. $(2007,2009)$ found abnormal reward-mediated striatal activation, and Roberts et al. (2007) noticed reduced flexibility associated with decreased activation in the striatum and associated areas (Zastrow et al., 2009). Similarly, Lopez et al. (2008) showed a bias towards focusing on detail at the expense of seeing the general picture (poor central coherence), and Zucker et al. (2007) and Harrison et al. (2009) found problems in social cognition and dysfunctional emotional regulation.

\section{Integrative models of anorexia nervosa}

Beyond the above enumeration of different factors that contribute to AN pathogenesis, some authors have proposed comprehensive models that combine some of these factors. Rather than an exhaustive presentation, present a representative sample of such models, each chosen because they exemplify a developmental and/or integrative approach to AN.

In 1993, Garner explored AN as a multifactorial disorder, and proposed a model with predisposing, precipitating and perpetuating factors. Individual predisposition comprises 
psychological factors (depression, anxiety, personality disorder, cognitive and emotional deficits, body image and psychological and physical trauma), as well as genetic vulnerability, constitutional and physical vulnerability and neuroendocrine and metabolic abnormalities. The two other broad classes of predisposing factors are familial and cultural predisposition. Garner focused on body dissatisfaction and effects of dieting on self-esteem and self-control. Perpetuating factors were associated with the psychological and physical effects of starvation and with interactions with the other factors.

In 2004, Jacobi et al. focused on risk factors associated with ED and proposed a graphical, timeline (from birth to onset) representation of ED, especially AN. Although the authors adopted a developmental perspective, they nevertheless specified that "the timelines are not intended to illustrate a causal pathway to ED, as combinations and interactions between risk factors was not the focus of the review". In addition to this and other findings about the age of onset of risk factors, these authors proposed fixed markers, variable markers, and retrospective correlates for AN from cross-sectional studies, both in relation to the timeline (from birth to AN onset) and according to potency of each factor (from not specified, to low, medium and high). As high potency factors, Jacobi et al. found female gender (genetic fixed marker) and high-levels of exercise. Medium factors were feeding/gastro-intestinal problems, sleep pattern difficulties in infancy, anxious parenting (from birth to 5 years), obsessive-compulsive personality disorder (from 5 to 18 years), perfectionism and negative self-evaluation (approximately 15 years). Following this innovative presentation, the authors addressed the complexity of risk factor status and emphasized the importance of the precedence of the factor to the disorder. In brief, Jacobi et al. examined some results in light of the type of population studied (community, clinical, or subclinical samples), the age or gender of patients, and the developmental or whole lifespan period, and they stressed the potential differences between risk factors associated with partial or full syndromes. Without proposing an integrative approach of AN, Jacobi et al. contributed to better comprehension of the complexity of risk factors, and they opened the way for their integration into a developmental model of AN. 
In 2010, Treasure et al. published an article in the Lancet. They approached ED and AN as "biologically based forms of severe mental illnesses," and they drew attention to the pathogenesis of AN and several associated risk factors: genetic factors, biological factors, environmental context, and a brief exploration of the interactions between the environment and individual biology. The authors did not develop a model as such, but they instead focused on interactions between these risk factors in different phases of AN.

In 2011, Hasan and Hasan proposed a unified neurological view of AN. They considered that comprehensive perspectives about AN were shifting from the bio-psycho-social model to the biological model. These authors considered the dysfunction of various neuropeptides in both the central nervous system (CNS) and in the peripheral nervous system (PNS). Hasan and Hasan attempted an integrative approach to propose a more holistic and complete model for AN. They focused on corticotrophin-releasing factor (CRF) as the key mediator of the hypothalamo-pituitary-adrenal (HPA) axis, on opioid peptides as the addictive component of $\mathrm{AN}$, and on leptin (role in suppressing appetite) and ghrelin. All of these neuropeptides were presented as operating collectively in the HPA axis, and a very detailed and exhaustive diagram was drawn to represent the key pathways involved in the spectrum of physiological and psychological symptoms of AN. The discussion focused on links between physiological abnormalities (CRF, HPA, ...) and associated symptoms through direct or complex pathways. For instance, starvation inhibits the noradrenergic stimulation of $\mathrm{CRF}$, leading to a depressed locus coeruleus (role in stress regulation, mediation of CRF through the action of opioids) (Hasan and Hasan, 2011). Nevertheless, the locus coeruleus (LC) is most likely also activated by the stress associated with AN. In other words, the effect of the LC could be biphasic and the release of noradrenaline could be intermittent. Another example concerned pathways for fear conditioning. Authors proposed that fear and its neurophysiology in AN could be understood by way of three distinct pathways: CRF, cortisol, and ghrelin. Finally, Hasan and Hasan (2011) recalled that starvation alone does not necessarily imply AN, as to moderate a too evident relationship between physiological abnormalities and their clinical correlations. 
The same year, Herpertz-Dahlmann et al. proposed a complex developmental model, partially supported by the contributions of Kaye et al. (2009), for the time course and phenomenology of AN. This etiological model explored AN pathogenesis from the prenatal period to adulthood and proposed developmental pathways including genetic factors, prenatal factors, traits and cognitive styles in childhood, the role of puberty and the effects of starvation on the brain. The authors focused on the consequences of starvation (which induces a cascade of pathophysiological reactions) and on its sequelae. Potential impact on the developing adolescent brain was explored in terms of the consequences of "biological scars".

In 2012, Nunn et al. proposed an empirically based hypothesis of noradrenergic dysregulation in AN. Without denying the important role that serotonin may play in AN, Nunn et al. considered that this neurotransmitter did not explain the wide range of clinical features in AN. The noradrenergic dysregulation hypothesis was supported by an explicative figure with ten interactive parameters. According to this model, these components, in cascade, lead to AN development. They include a specific noradrenergic genetic profile, epigenetic factors, noradrenergic dysregulation, sympathetic overactivity, impaired neuroplasticity, reduction of cerebral blood flow, insula dysfunction, homunculus dysfunction, distorted body image and precursor depletion. The overall interactions of all of these components could contribute to the development of AN. More precisely, Nunn et al. remarked that noradrenaline had multiple functions, such as regulating sympathetic arousal (and therefore levels of anxiety), mediating neuroplasticity or regulating central blood flow (as previously noted, particularly in the temporal region). In addition, Nunn et al. hypothesized that, as a result of noradrenergic dysregulation and its impairment of neuroplasticity, the insula may dysfunction and play a major role in AN. They recall the wide range of functions of the insular cortex, such as interoception, bodily representation and visual perception (marked dysregulation in $A N$ ), perception of pain and taste, regulation of empathy and disgust, regulation of the autonomic nervous system or integration of thoughts and feelings. These authors discussed the implication of other neurotransmitter systems in $\mathrm{AN}$ and remarked that none of them 
operated in isolation. For instance, there are persistent alterations in the serotonergic system even after recovery, and abnormalities of this system could be trait-related, emphasizing the lack of any integrating hypothesis implicating this system (Kaye et al., 2005). Nunn et al. suggested that dopamine (a precursor of noradrenaline) "an intact reinforcement reward system sustained the restrictive behaviors through partial, intermittent reinforcement of food restriction, paired with anxiety reduction". All of these data question the established role of abnormalities in dopamine-mediated reward systems and serotonin-regulated neuromodulation systems as causal mechanisms in AN (Nunn et al., 2012), and they also point to a genuine role of noradrenergic dysfunction in AN, even if an isolated causal role is improbable. Along these lines, the authors suggested other potential neurotransmitter implications in AN and recalled different anomalies involving GABA, acetylcholine, dynorphin or estrogen associated with $\mathrm{AN}$, and also the fact that the noradrenergic, anticholinergic, serotonergic and glutamate systems mediate neuroplasticity (Marzo et al., 2009, Gu, 2002).

\section{Conclusions}

Anorexia nervosa is associated with a lifelong trajectory and the continued appearance of multiple risk factors over the entire disease time course, including a vulnerability period, puberty, post-pubertal years, adolescence, age of onset, chronic illness and illness course. Indeed, anorexia nervosa occurs mainly during an intense and limited upside-down period for the individual concerned, adolescence. Most precipitating risk factors, isolated or combined, that progressively appear in the prelude to the onset of $\mathrm{AN}$, are generally asymptomatic and sometimes transient. One major difficulty is to differentiate the numerous risk factors and other potential premorbid alterations from typical developmental reorganizations and to individualize their actual causal role. Likewise, another difficulty resides in associating a physiological abnormality with a functional impairment and in considering that physiological restorations may be synonymous with recovery. Even if some 
brain regions, such as the temporal lobes, or structures, such as the insula, are of interest, results are still debated and need further longitudinal exploration. Beyond the complexity of risk factors, the acute phase also reveals a complex network of pathways that contribute both to the worsening and to the persistence of $\mathrm{AN}$ through a progressive, interactive combination of physiological and psychological processes. Once again, time (i.e., the duration of illness) is a major parameter. Indeed, on the one hand, AN is time-mediated because of its temporal pathogenesis, and, on the other hand, the consequences of $\mathrm{AN}$ (mainly during the chronic phase) interfere with or halt individual development as if time was "suspended". A growing number of studies have been conducted in light of new integrative and developmental models, from genetic considerations to comprehensive psychological approaches. An interesting therapeutic application of this research field could concern potential adjustments to temporal parameters and features of care strategies. In other words, better prognoses involve the right care at the right moment for optimal efficacy and to prevent the chronic evolution of the illness. In summary, we can raise the following issue: should anorexia nervosa be considered as a developmental pathology or as a pathology of development? 


\section{References}

Ackard, D.M., Fulkerson, J.A., Neumark-Sztainer, D., 2007. Prevalence and utility of DSM-IV eating disorder diagnostic criteria among youth. Int. J. Eat. Disord. 40(5), 409-417.

American Psychiatric Association, 2000. Diagnostic and Statistical Manual of Mental Disorders, fourth ed. American Psychiatric Association, Washington, DC (text revision).

Bailer, U.F., Price, J.C., Meltzer, C.C., Mathis, C.A., Frank, G.K., Weissfeld, L., McConaha, C.W., Henry, S.E., Brooks-Achenbach, S., Barbarich, N.C., Kaye, W.H., 2004. Altered 5-HT(2A) receptor binding after recovery from bulimia-type anorexia nervosa : relationships to harm avoidance and drive for thinness. Neuropsychopharmacology 29 (6), 1143-1155.

Bailer, U.F., Frank, G.K., Henry, S.E., Price, J.C., Meltzer, C.C., Becker, C., Ziolko, S.K., Mathis, C.A., Wagner, A., Barbarich-Marsteller, N.C., Putnam, K., Kaye, WH., 2007. Serotonin transporter binding after recovery from eating disorders. Psychopharmacology 195 (3), 315-324.

Baker, J.H., Mazzeo, S.E., Kendler, K.S., 2007. Association between broadly defined bulimia nervosa and drug use disorders: common genetic and environmental influences. Int. J. Eat. Disord. 40 (8), 673-678.

Beato-Fernández, L., Rodríguez-Cano, T., García-Vilches, I., García-Vicente, A., Poblete-García, V., Castrejon, A.S., Toro, J., 2009. Changes in régional cérébral blood flow after body image exposure in eating disorders. Psychiatry Res. 171 (2), 129-137.

Bergen, A.W., Van den Bree, M.B., Yeager, M., Welch, R., Ganjei, J.K., Haque, K., Bacanu, S., Berrettini, W.H., Grice, D.E., Goldman, D., Bulik, C.M., Klump, K., Fichter, M., Halmi, K., Kaplan, A., Strober, M., Treasure, J., Woodside, B., Kaye, W.H., 2003. Candidate genes for anorexia nervosa in the 1p33-36 linkage region: Serotonin 1D and delta opioid receptor loci exhibit significant association to anorexia nervosa. Mol. Psychiatry 8 (4), 397-406. 
Bloomfield, F.H., Oliver, M.H., Hawkins, P., Campbell, M., Phillips, D.J., Gluckman, P.D., Challis, J.R., Harding, J.E., 2003. A periconceptional nutritional origin for noninfectious preterm birth. Science 300 (5619), 606.

Boghi, A., Sterpone, S., Sales, S., D'Agata, F., Bradac, G.B., Zullo, G., Munno, D., 2011. In vivo evidence of global and focal brain alterations in anorexia nervosa. Psychiatry Res. 192 (3), 154-159.

Brambilla, F., Monteleone, P., Bortolotti, F., Dalle Grave, R., Todisco, P., Favaro, A., Santonastaso, P., Ramacciotti, C., Paoli, R., Maj, M., 2003. Persistent amenorrhoea in weight-recovered anorexics: psychological and biological aspects. Psychiatry Res. 118 (3), 249-257.

Bredella, M.A., Misra, M., Miller, K.K., Klibanski, A., Gupta, R., 2008. Trabecular structure analysis of the distal radius in adolescent patients with anorexia nervosa using ultra high resolution flat panel based volume CT. J. Musculoskelet Neuronal Interact. 8 (4), 315.

Bürhen, K., Mainz, V., Herpertz-Dahlmann, B., Schäfer, K., Kahraman-Lanzerath, B., Lente, C., Konrad K., 2012. Cognitive flexibility in juvenile anorexia nervosa patients before and after weight recovery. J. Neural Transm. (Epub ahead of print)

Carter, J.C., Blackmore, E., Sutandar-Pinnock, K., Woodside, D.B., 2004. Relapse in anorexia nervosa: a survival analysis. Psychol. Med. 34 (4), 671-679.

Castro-Fornieles, J., Bargallo, N., Lazaro, L., Andrés, S., Falcon, C., Plana, M.T., Junqué, C., 2009. A cross-sectional and follow-up voxel-based morphometric MRI study in adolescent anorexia nervosa. J. Psychiatr. Res. 43 (3), 331-340.

Castro-Fornieles, J., Caldú, X., Andrés-Perpiñá, S., Lázaro, L., Bargalló, N., Falcón, C., Plana, M.T., Junqué, C., 2010. A cross-sectional and follow-up functional MRI study with a working memory task in adolescent anorexia nervosa. Neuropsychologia 48 (14), 4111-4116. 
Chowdhury, U., Gordon, I., Lask, B., Watkins, B., Watt, H., Christie, D., 2003. Early-onset anorexia nervosa: is there evidence of limbic system imbalance? Int. J. Eat. Disord. 33 (4), 388-396.

Chui, H.T., Christensen, B.K., Zipursky, R.B., Richards, B.A., Hanratty, M.K., Kabani, N.J., Mikulis, D.J., Katzman., 2008. Cognitive function and brain structure in females with a history of adolescentonset anorexia nervosa. Pediatrics 122 (2), 426-427.

Cnattingius, S., Hultman, C., Dahl, M., Sparen, P., 1999. Very preterm birth, birth trauma, and the risk of anorexia nervosa among girls. Arch. Gen. Psychiatry 56 (7), 634-638.

Devlin, B., Bacanu, S., Klump, K., Bulik, C.M., Fichter, M., Halmi, K., Kaplan, A.S., Strober, M., Treasure, J., Woodside, D.B., Berrettini, W.H., Kaye, W.H., 2002. Linkage analysis of anorexia nervosa in incorporating behavioral covariates. Hum. Mol. Genet. 11 (6), 689-696.

Dominguez, J., Goodman, L., Sen Gupta, S., Mayer, L., Etu, S.F., Walsh, B.T., Wang, J., Pierson, R., Warren, M.P., 2007. Treatment of anorexia nervosa is associated with increases in bone mineral density, and recovery is a biphasic process involving both nutrition and return of menses. Am. J. Clin. Nutr. 86(1), 92-99.

Ellison, Z., Foong, J., Howard, R., Bullmore, E., Williams, S., Treasure, J., 1998. Functional anatomy of calorie fear in anorexia nervosa. Lancet 352 (9135), 1192.

Favaro, A., Tenconi, E., Santonastaso, P., 2006. Perinatal factors and the risk of developing anorexia nervosa and bulimia nervosa. Arch. Gen. Psychiatry 63 (1), 82-88.

Fox, E.A., 2008. Purdue Ingestive Behavior Research Center symposium 2007: influences on eating and body weight over the lifespan-childhood and adolescence. Physiol. Behav. 94 (1), 1-7. 
Frampton, I., Watkins, B., Gordon, I., Lask, B., 2011. Do abnormalities in regional cerebral blood flow in anorexia nervosa resolve after weight restoration? Eur. Eat. Disord. Rev. 19 (1), 55-58.

Frank, G.K., Kaye, W.H., Meltzer, C.C., Price, J.C., Greer, P., McConaha, C., Skovira, K., 2002. Reduced 5-HT2A receptor binding after recovery from anorexia nervosa. Biol. Psychiatry 52 (9), 896 906.

Frank, G.K., Bailer, U.F., Meltzer, C.C., Price, J.C., Mathis, C.A., Wagner, A., Becker, C., Kaye, W.H., 2007. Regional cerebral blood flow after recovery from anorexia nervosa or bulimia nervosa. Int. J. Eat. Disord. 40 (6), 488-492.

Friederich HC., Herzog W., 2011. Cognitive-behavioral flexibility in anorexia nervosa. Curr. Top. Behav. Neurosci. 6, 111-123.

Garner, D.M., 1993. Pathogenesis of anorexia nervosa. Lancet 341 (8861), 1631-1635.

Gaudio, S., Nocchi, F., Franchin, T., Genovese, E., Cannatà, V., Longo, D., Fariello, G., 2011. Gray matter decrease distribution in the early stages of Anorexia Nervosa restrictive type in adolescents. Psychiatry Res. 191 (1), 24-30.

Gérard, N., Pieters, G., Goffin, K., Bormans, G., Van Laere, K., 2011. Brain type 1 cannabinoid receptor availability in patients with anorexia and bulimia nervosa. Biol. Psychiatry 70 (8), 777-784.

Gillberg, I.C., Billstedt, E., Wentz, E., Anckarsäter, H., Rastam, M., Gillberg, C., 2010. Attention, executive functions, and mentalizing in anorexia nervosa eighteen years after onset of eating disorder. J. Clin. Exp. Neuropsychol. 32 (4), 358-365.

Gordon, I., Lask, B., Bryant-Waugh, R., Christie, D., Timimi, S., 1997. Childhood-onset anorexia nervosa: towards identifying a biological substrate. Int. J. Eat. Disord. 22 (2), 159-165. 
Gordon, R.A., 2001. Eating disorders East and West: A culture-bound syndrome unbound. In M. Nasser, MA Katzman, RA Gordon (Eds). Eating disorders and culture in transition, BrunnerRoutledge, New York.

Grice, D.E., Halmi, K.A., Fichter, M.M., Strober, M., Woodside, D.B., Treasure, JT., Kaplan, A.S., Magistretti, P.J., Goldman D., Bulik, C.M., Kaye, W.H., Berrettini, W.H., 2002. Evidence for a susceptibility gene for anorexia nervosa on chromosome 1. Am. J. Hum. Genet. 70 (3), 787-792.

Grinspoon, S., Baum, H., Lee, K., Anderson, E., Herzog, D., Klibanski, A., 1996. Effects of short-term recombinant human insulin-like growth factor I administration on bone turnover in osteopenic women with anorexia nervosa. J. Clin. Endocrinol. Metab. 81 (11), 3864-3870.

Grinspoon, S., Thomas, E., Pitts, S., Gross, E., Mickley, D., Miller, K., Herzog, D., Klibanski, A., 2000. Prevalence and predictive factors for regional osteopenia in women with anorexia nervosa. Ann. Intern. Med. 133 (10), 790-794.

Gu, Q., 2002. Neuromodulatory transmitter systems in the cortex and their role in cortical plasticity. Neuroscience 111 (4), 815-835.

Gwirtsman, H.E., Kaye, W.H., George, D.T., Jimerson, D.C., Ebert, M.H., Gold, P.W., 1989. Central and peripheral ACTH and cortisol levels in anorexia nervosa and bulimia. Arch. Gen. Psychiatry 46 (1), 61-69.

Hadigan, C.M., Anderson, E.J., Miller, K.K., Hubbard, J.L., Herzog, D.B., Klibanski, A., Grinspoon, S.K., 2000. Assessment of macronutrient and micronutrient intake in women with anorexia nervosa. Int. J. Eat. Disord. 28 (3), 284-292.

Harrison, A., Sullivan, S., Tchanturia, K., Treasure, J., 2009. Emotion recognition and regulation in anorexia nervosa. Clin. Psychol. Psychother. 16 (4), 348-356. 
Hasan, T.F., Hasan, H., 2011. Anorexia nervosa: A unified neurological perspective. Int. J. Med. Sci. 8 (8), 679-703.

Helder, S.G., Collier, D.A., 2011. The genetics of eating disorders. Curr. Top. Behav. Neurosci. 6, 157175.

Hermann, T., Siega-Ritz, A., Hobel, C., Aurora, C., Dunkel-Schetter, C., 2001. Prolonged periods without food intake during pregnancy increase risk for elevated maternal corticotropin-releasing hormone concentrations. Am. J. Obstet. Gynecol. 185 (2), 402-412.

Herpertz-Dahlmann, B., Seitz, J., Konrad, K., 2011. Aetiology of anorexia nervosa: from a "psychosomatic family model" to a neuropsychiatric disorder?. Eur. Arch. Psychiatry Clin. Neurosci. 261 (S2), 177-181.

Herzog, W., Minne, H., Deter, C., Leidig, G., Schellberg, D., Wuster, C., Gronwald, R., Sarembe, E., Kroger, F., Bergmann, G., Petzold, E., Hahn, P., Schepank, H., Ziegler, R., 1993. Outcome of bone mineral density in anorexia nervosa patients 11.7 years after first admission. J. Bone Miner. Res. 8 (5), 597-605.

Hoek, H.W., 1993. Review of the epidemiological studies of eating disorders. Int. Rev. Psychiatry 5, $61-74$.

Hoek, H.W., Hoeken, D., 2003. Review of the prevalence and incidence of eating disorders. Int. J. Eat. Disord. 34 (4), 383-396.

Hudson, J.I., Lalonde, J.K., Berry, J.M., Pindyck, L.J., Bulik, C.M., Crow, S.J., McElroy, S.L., Laird, N.M., Tsuang, M.T., Walsh, B.T., Rosenthal, N.R., Pope, H.G. Jr., 2007. Binge-eating disorder as a distinct familial phenotype in obese individuals. Arch. Gen. Psychiatry 63 (3), 313-319. 
Jacoangeli, F., Masala, S., Staar Mezzasalma, F., Fiori, R., Martinetti, A., Ficoneri, C., Novi, B., Pierangeli, S., Marchetti, G., Simonetti, G., Bollea, M.R., 2006. Amenorrhea after weight recover in anorexia nervosa: role of body composition and endocrine abnormalities. Eat. Weight Disord. 11 (1), 20-26.

Jacobi, C., Hayward, C., de Zwaan, M., Kraemer, HC., Agras, WS., 2004. Coming to terms with risk factors for eating disorders: Application of risk terminology and suggestions for a general taxonomy. Psychol. Bull. 130 (1), 19-65.

Jáuregui-Loreba, I., 2011. Neuroimaging in eating disorders. Neuropsy. Dis. Treat. 7, 577-584.

Jiménez-Bonilla, J.F., Quirce, R., Banzo, I., Martínez-Rodríguez, I., Sainz-Esteban, A., Portilla, H., Carril, J.M., 2009. Temporal hypoperfusion assessed by cerebral blood flow SPECT and long-term clinical outcome in patients with eating disorders. Clin. Nucl. Med. 34 (11), 768-772.

Johnson, J.G., Cohen, P., Kasen, S., Brook, J.S., 2002. Eating disorders during adolescence and the risk of physical and mental disorders during early adulthood. Arch. Gen. Psychiatry 59 (6), 545-552.

Joos, A.A., Perlov, E., Büchert, M., Hartmann, A., Saum, B., Glauche, V., Freyer, T., Weber-Fahr, W., Zeeck, A., Tebartz van Elst, L., 2010. Magnetic resonance spectroscopy of the anterior cingulated cortex in eating disorders. Psychiatry Res. 191 (3), 196-200.

Kaye, W.H., Bailer, U.F., Frank, G.K., Wagner, A., Henry, S.E., 2005. Brain imaging of serotonin after recovery from anorexia nervosa and bulimia nervosa. Physiol. Behav. 86 (1-2), 15-17.

Kaye, W,H., Fudge, J.L., Paulus, M., 2009. New insights into symptoms and neurocircuit function of anorexia nervosa. Nat. Rev. Neurosci. 10 (8), 573-584.

Kaye, W.H., Bailer, U.F., 2011. Understanding the neural circuitry of appetitive regulation in eating disorders. Biol. Psychiatry 70 (8), 704-705. 
Keel, P.K., Klump, K.L., 2005. Are eating disorders culture-bound syndromes? Implications for conceptualizing their etiology. Psychol. Bull. 129 (5), 747-769.

Kessler, R.C., Berglund, P., Chiu, W.T., Demler, O., Heeringa, S., Hiripi, E., Jin, R., Pennell, B.E., Walters, E.E., Zaslavsky, A., Zheng, H., 2004. The US National Comorbidity Survey Replication (NCSR): Design and field procedures. Int. J. Methods. Psychiatr. Res. 13 (2), 69-92.

Kessler, R.C., Chiu, W.T., Dernier, O., Walters, E.E., 2005. Prevalence, severity, and comorbidity of 12-month DSM-IV disorders in the National Comorbidity Survey Replication. Arch. Gen. Psychiatry 62 (6), 617-627.

Klump, K.L., Miller, K.B., Keel, P.K., McGue, M., lacono, W.G., 2001. Genetic and environmental influences on anorexia nervosa syndromes in a population-based twin sample. Psychol. Med. 31 (4), 737-740.

Komatzu, H., Nagamitsu, S., Ozono, S., Yamashita, Y., Ishibashi, M., Matsuishi, T., 2010. Regional cerebral blood flow changes in early-onset anorexia nervosa before and after weight gain. Brain Dev. 32 (8), 625-630.

Kortegaard, L.S., Hoerder, K., Joergensen, J., Gillberg, C., Kyvik, K.O., 2001. A preliminary population-based twin study of self-reported eating disorder. Psychol. Med. 31 (2), 361-365.

Lask, B., Gordon, I., Christie, D., Frampton, I., Chowdhury, U., Watkins, B., 2005. Functional neuroimaging in early-onset anorexia nervosa. Int. J. Eat. Disord. 37, S49-S51.

Lawson, E.A., Misra, M., Meenaghan, E., Rosenblum, L., Donoho, D., Herzog, D., Klibanski A., Miller, K.K., 2009. Adrenal glucocorticoid and androgen precursor dissociation in anorexia nervosa. J. Clin. Endocrinol. Metab. 94 (4), 1367-1371. 
Lawson, E.A., Miller, K.K., Bredella, M.A., Phan, C., Misra, M., Meenaghan, E., Rosenblum, L., Donoho, D., Gupta, R., Klibanski A., 2010. Hormone predictors of abnormal bone microarchitecture in women with anorexia nervosa. Bone 46 (2), 458-463.

Liao, P.C, Uher, R., Lawrence, N., Treasure, J., Schmidt, U., Campbell, I.C., Collier, D.A., Tchanturia, K., 2009. An examination of decision making in bulimia nervosa. J. Clin. Exp. Neuropsychol. 31 (4), 455-461.

Levine, M., 1998. Prevention of eating problems with elementary children. US: USA Today.

Lopez, C., Tchanturia, K., Stahl, D., Treasure, J., 2008. Central coherence in eating disorders: a systematic review. Psychol. Med. 38 (10), 1393-1404.

Lucas AR., Crowson CS., O'Fallon WM., Melton LJ., 1999. The ups and downs of anorexia nervosa. Int. J. Eat. Disord. 26 (4), 397-405.

Marsh, R., Maia, T.V., Peterson, B.S., 2009. Functional disturbances within frontostriatal circuits across multiple childhood psychopathologies. Am. J. Psychiatry 166 (6), 664-674.

Marzo, A., Bai, J., Otani, S., 2009. Neuroplasticity regulation by noradrenaline in mammalian brain. Curr. Neuropharmacol. 7 (4), 286-295.

Mc Cormick, L.M., Ziebell, S., Nopoulos, P., Cassell, M., Andreasen, N.C., Brumm, M., 2006. Anterior cingulated cortex: an MRI-based parcellation method. Neuroimage 32 (3), 1167-1175.

Mc Cormick, L.M., Keel, P.K., Brumm, M.C., Bowers, W., Swayze, V., Andersen, A., Andreasen, N.C., 2008. Implications of starvation-induced change in right dorsal anterior cingulated volume in anorexia nervosa. Int. J. Eat. Disord. 41 (7), 602-610. 
McKnight investigators, 2003. Risk factors for the onset of eating disorders in adolescent girls: Results of the McKnight longitudinal risk factor study. Am. J. Psychiatry 160 (2), 248-254.

Miller, K.K., Grinspoon, S.K., Ciampa, J., Hier, J., Herzog, D., Klibanski, A., 2005. Medical findings in outpatients with anorexia nervosa. Arch. Intern. Med. 165 (5), 561-566.

Miller, K.K., Lee, E.E., Lawson, E.A., Misra, M., Minihan, J., Grinspoon, S.K., Gleysteen, S., Mickley, D., Herzog, D., Klibanski, A., 2006. Determinants of skeletal loss and recovery in anorexia nervosa. J. Clin. Endocrinol. Metab. 91 (8), 2931-2937.

Miller, K.K., Wexler, T.L., Zha, A.M., Lawson, E.A., Meenaghan, E.M., Misra, M., Binstock, A.B., Herzog, D., Klibanski, A., 2007. Androgen deficiency: association with increased anxiety and depression symptom severity in anorexia nervosa. J. Clin. Psychiatry 68 (6), 959-965.

Miller, K.K., 2011. Endocrine dysregulation in anorexia nervosa Update. J. Clin. Endocrinol. Metab. 96 (10), 2939-2949.

Misra, M., Aggarwal, A., Miller, K.K., Almazan, C., Worley, M., Soyka, L.A., Herzog, D., Klibanski, A., 2004. Effects of anorexia nervosa on clinical, hematologic, biochemical, and bone density parameters in community-dwelling adolescent girls. Pediatrics 114 (6), 1574-1583.

Misra, M., Prabhakaran, R., Miller, K.K., Tsai, P., Lin, A., Lee, N., Herzog, D., Klibanski, A., 2006. Role of cortisol in menstrual recovery in adolescent girls with anorexia nervosa. Pediatr. Res. 59 (4), 598603.

Mohr, H.M., Zimmermann, J., Röder, C., Lenz, C., Overbeck, G., Grabhorn, R., 2010. Separating two components of body image in anorexia nervosa using fMRI. Psychol. Med. 40 (9), 1519-1529. 
Mühlau, M., Gaser, C., Ilg, R., Conrad, B., Leibl, C., Cebulla, M.H., Backmund, H., Gerlinghoff, M., Lommer, P., Schnebel, A., Wohlschläger, A.M., Zimmer, C., Nunnemann, S.I., 2007. Gray matter decrease of the anterior cingulate cortex in anorexia nervosa. Am. J. Psychiatry 164 (12), 1850-1857.

Nakazato, M., Tchanturia, K., Schmidt, U., Campbell, I.C., Treasure, J., Collier, D.A., Hashimoto, K., Iyo, M.., 2009. Brain-derived neurotrophic factor (BDNF) and set-shifting in currently ill and recovered anorexia nervosa (AN) patients. Psychol. Med. 39 (6), 1029-1035.

Naruo, T., Nakabeppu, Y., Sagiyama, K., Munemoto, T., Homan, N., Deguchi, D., Nakajo, M., Nozoe, S., 2000. Characteristic regional cerebral blood flow patterns in anorexia nervosa patients with binge/purge behavior. Am. J. Psychiatry 157 (9), 1520-1522.

Neufgang, S., Specht, K., Hausmann, M., Güntürkün, O., Herpertz-Dahlmann, B., Fink, G.R., Konrad, K., 2009. Sex differences and the impact of steroid hormones on the developing human brain. Cereb. Cortex 19 (2), 464-473.

Norre J., Vandereycken W., Gordts S., 2001. The management of eating disorders in a fertility clinic: Clinical guidelines. J. Psychosom. Obstet. Gynaecol. 22 (2), 77-81.

Nozoe, S., Naruo, T., Nakabeppu, Y., Soejima, Y., Nakajo, M., Tanaka, H., 1993. Changes in regional cerebral blood flow in patients with anorexia nervosa detected through single photon emission tomography imaging. Biol. Psychiatry 34 (8), 578-580.

Nozoe, S., Naruo, T., Yonekura, R., 1993. Comparison of regional cerebral blood flow in patients with eating disorders. Brain Res. Bull. 36 (3), 251-255.

Nunn, K., Frampton, I., Lask, B., 2012. Anorexia nervosa - A noradrenergic dysregulation hypothesis. Med. Hypothesis 78 (5), 580-584. 
Papadopoulos, F.C., Ekbom, A., Brandt, L., Ekselius, L., 2009. Excess mortality, causes of death and prognostic factors in anorexia nervosa. Br. J. Psychiatry 194 (1), 10-17.

Pinheiro, A.P., Bulik, C.M., Thornton, L.M., Sullivan, P.F., Root, T.L., Bloss, C.S., Berrettini, W.H., Schork, N.J., Kaye, W.H., Bergen, A.W., Magistretti, P., Brandt, H., Crawford, S., Crow, S., Fichter, M.M., Goldman, D; Halmi, K.A., Johnson, C., Kaplan, A.S., Keel, P.K., Klump, K.L., La Via, M., Mitchell, J.E., Strober, M., Rotondo, A., Treasure, J., Woodside, D.B., 2010. Association study of 182 candidate genes in anorexia nervosa. Am. J. Med. Genet. Part B 153B, 1070-1080.

Rastam, M., 1992. Anorexia nervosa in 51 Swedish adolescents: premorbid problems and comorbidity. J. Am. Acad. Child. Adolesc. Psychiatry 31 (5), 819-829.

Ribasés, M., Gratacòs, M., Fernández-Aranda, F., Bellodi, L., Boni, C., Anderluh, M., Cristina Cavallini, M., Cellini, E., Di Bella, D., Erzegovesi, S., Foulon, C., Gabrovsek, M., Gorwood, P., Hebebrand, J., Hinney, A., Holliday, J., Hu, X., Karwautz, A., Kipman, A., Komel, R., Nacmias, B., Remschmidt, H., Ricca, V., Sorbi, S., Tomori, M., Wagner, G., Treasure, J., Collier, D.A., Estivill, X., 2005. Association of BDNF with restricting anorexia nervosa and minimum body mass index: a familybased association study of eight European populations. Eur. J. Hum. Genet. 13 (4), 428-434.

Rigotti, N.A., Neer, R.M., Skates, S.J., Herzog, D., Nussbaum, S.R., 1991. The clinical course of osteoporosis in anorexia nervosa. A longitudinal study of cortisol bone mass. JAMA 265 (9), 11331138.

Roberts, M.E., Tchanturia, K., Stahl, D., Southgate, L., Treasure, J., 2007. A systematic review and meta-analysis of set-shifting ability in eating disorders. Psychol. Med .37 (8), 1075-1084.

Rodin, J., Silberstein, L., Striegel-Moore, R., 1985. Women and weight: A normative discontent. In T. Sonderegger (Ed.), Psychology and gender: Nebraska Symposium on Motivation, Lincoln: University of Nebraska Press, 267-307. 
Schmidt, U., Treasure, J., 2006. Anorexia nervosa: valued and visible. A cognitive-interpersonal maintenance model and its implications for research and practice. Br. J. Clin. Psychol. 45 (3), 1-25.

Shoebridge, P., Gowers, S.G., 2000. Parental high concern and adolescent-onset anorexia. A casecontrol study to investigate direction of causality. Br. J. Psychiatry 176, 132-137.

Soyka, L.A., Misra, M., Frenchman, A., Miller, K.K., Grinspoon, S., Schoenfeld, D.A., Klibanski, A., 2002. Abnormal bone mineral accrual in adolescent girls with anorexia nervosa. J. Clin. Endocrinol. Metab. 87 (9), 4177-4185.

Steinhausen, H.C., 2002. The outcome of anorexia nervosa in the $20^{\text {th }}$ Century. Am. J. Psychiatry 159 (8), 1284-1293.

Strigel-Moore RH., Bulik CM., 2007. Risk factors for eating disorders. Am. Psychol. 62 (3), 181-198.

Suda, M., Uehara, T., Fukuda, M., Sato, T., Kameyama, M., Mikuni, M., 2010. Dieting tendency and eating behavior problems in eating disorder correlate with right frontotemporal and left orbitofrontal cortex: a near-infrared spectroscopy study. J. Psychiatr. Res. 44 (8), 547-555.

Treasure J., Claudino AM., Zucker N., 2010. Eating disorders. Lancet 375 (9714), 583-593.

Uher, R., Brammer, M.J., Murphy, T., Campbell, I.C., Ng, V.W., Williams, S.C., Treasure, J., 2003. Recovery and chronicity in anorexia nervosa: brain activity associated with differential outcomes. Biol. Psychiatry 54 (9), 934-942.

Uher, R., Murphy, T., Brammer, M.J., Dalgleish, T., Phillips, M.L., Ng, V.W., Andrew, C.M., Williams, S.C., Campbell, I.C., Treasure, J. 2004. Medial prefrontal cortex activity associated with symptom provocation in eating disorders. Am. J. Psychiatry 161 (7), 1238-1246. 
Van den Eynde, F., Treasure, J., 2009. Neuroimaging in eating disorders and obesity: implications for research. Child Adolesc. Psychiatr. Clin. N. Am. 18 (1), 95-115.

Van den Eynde, F., Suda, M., Broadbent, H., Guillaume, S., Van den Eynde, M., Steiger, H., Israel, M., Berlim, M., Giampietro, V., Simmons, A., Treasure, J., Campbell, I., Schmidt, U., 2012. Structural magnetic resonance imaging in eating disorders: a systematic review of voxel-based morphometry studies. Eur. Eat. Disord. Rev. 20 (2), 94-105.

Wade, T., Bulik, C.M., Neale, M., Kendler, K.S., 2000. Anorexia nervosa and major depression: Shared genetic and environmental risk factors. Am. J. Psychiatry 157 (3), 469-471.

Wagner, A., Greer, P., Bailer, U.F., Frank, G.K., Henry, S.E., Putman, K., Meltzer, C.C., Ziolko, S.K., Hoge, J., McConaha, C., Kaye, W.H., 2006. Normal brain tissue volumes after long-term recovery in anorexia and bulimia nervosa. Biol. Psychiatry 59 (3), 291-293.

Wagner, A., Aizenstein, H., Venkatraman, V.K., Fudge, J., May, J.C., Mazurkewicz, L., Frank, G.K., Bailer, U.F., Fischer, L., Nguyen, V., Carter, C., Putnam, K., Kaye, W.H., 2007. Altered reward processing in women recovered from anorexia nervosa. Am. J. Psychiatry 164 (12), 1842-1849.

Wagner, A., Aizenstein, H., Venkatraman, V.K., Fudge, J., May, J.C., Mazurkewicz, L., Frank, G.K., Bailer, U.F., Fischer, L., Nguyen, V., Carter, C., Putnam, K., Kaye, W.H., 2009. Altered striatal response to reward in bulimia nervosa after recovery. Int. J. Eat. Disord. 43 (4), 289-294.

Wang, K., Zhang, H., Bloss, CS., Duvvuri, V., Kaye, W.H., Schork, N.J., Berrettini, W., Hakonarson, H., Price Foundation Collaborative Group., 2010. Genome-wide association study on common SNPs and rare CNVs in anorexia nervosa. Mol. Psychiatry 16 (9), 949-959.

Waugh, E.J., Woodside, D.B., Beaton, D.E., Coté, P. Hawker, G.A., 2011. Effects of exercise on bone mass in young women with anorexia nervosa. Med. Sci. Sports Exerc. 43 (5), 755-763. 
Wentz, E., Gillberg, I.C., Anckarsäter, H., Gillberg, C., Rastam, M., 2009. Adolescent-onset anorexia nervosa: 18-year outcome. Br. J. Psychiatry 194 (2), 168-174.

Zastrow, A., Kaiser, S., Stippich, C., Walther, S., Herzog, W., Tchanturia, K., Belger, A., Weisbrod, M., Treasure, J., Friederich, H.C., 2009. Neural correlates of impaired cognitive-behavioral flexibility in anorexia nervosa. Am. J. Psychiatry 166 (5), 608-616.

Zucker, N.L., Losh, M., Bulik, C.M., LaBar, K.S., Piven, J., Pelphrey, K.A., 2007. Anorexia nervosa and autism spectrum disorders: guided investigation of social cognitive endophenotypes. Psychol. Bull. $133(6), 976-1006$. 